\title{
Th17: A New Player to be Considered in Tuberculosis Studies
}

\section{Cesar Pedroza-Roldán', Jeannette Barba² and Mario Alberto Flores-Valdez ${ }^{1 *}$}

${ }^{1}$ Center for Research and Assistance in Technology and Design of the State of Jalisco, AC, Medical and Pharmaceutical Biotechnology, Av normalistas 800, Col. Hills Normal, Guadalajara, Jalisco 44270, Mexico

${ }^{2}$ University of Guadalajara, University Center of Biological and Agricultural Sciences, Department of Public Health, Km 15.5 Guadalajara-Nogales, CP 44171, Zapopan, Jalisco, Mexico

Mycobacterium tuberculosis is the main causal agent of tuberculosis among people around the world. According to the World Health Organization (WHO) in its 2011 report, M. tuberculosis causes more than 1.4 million deaths around the world each year. Moreover, an increase of mortality has been observed among people co-infected with HIV. M. tuberculosis is transmitted after inhalation of contaminated droplets that are deposited in the distal alveoli, in this region, alveolar macrophages and dendritic cells ingest the bacteria [1]. One of the main functions of these cells is to destroy the pathogen by forming intracellular compartments such as the phagosome. However, M. tuberculosis has the ability to modulate phagosome maturation and in consequence is in some instances not eliminated.

For years, immunological studies have suggested that T-cell rather than B-cell mediated immunity is related with bacterial contention in animal models, and among infected people [1]. This bias can be justified by the fact that $\mathrm{T}$ cell mediated immunity is essential for containment of intracellular pathogens by several mechanisms. One of these mechanisms is related with CD4+ T helper cells. Studies in mice have suggested that an active CD4+ T helper cells 1 (Th1)-mediated response is essential for bacterial containment at the site of infection. This lineage of T cells is dependent of the presence of IL-12 (IL12p40/IL12p35, also named IL-12p70) that is mainly produced by antigen presenting cells such as Dendritic Cells (DCs), monocytes and macrophages; the last one, the principal host for M. tuberculosis. The secretion of IFN- $\gamma$ characterizes Th1 population that in concert with other Th1 cytokines activates macrophages to destroy the bacteria.

In recent years, a new $\mathrm{CD} 4+\mathrm{T}$ cells lineage emerged from investigations related with autoimmune diseases and inflammation, independent of IFN- $\gamma$, first reported in mouse models, called Th17. Interleukin 17 production (family composed of 6 members IL-17A-F) and lack of IFN- $\gamma$ secretion characterizes this T cell population. The presence of IL-23 (IL-12p40/IL-12p19) and small amounts of TGF- $\beta$ and IL- 6 are required for Th17 differentiation [2]. Evidence suggests that Th17 has a protective role when Th1-Th2 profiles are unable to act, for example against some extracellular pathogen and fungi.

Recent research has tried to determine the role of this new $\mathrm{T}$ $\mathrm{CD} 4+$ population during the infection with $\mathrm{M}$. tuberculosis. Evidences obtained thus far suggest that IL-17 is important during the first steps of infection. A mouse model deficient in IL-17 or IL-23 signaling is unable to control a high bacillary (103 CFU) dose infection with $\mathrm{M}$. tuberculosis when infection proceeds via intratracheal [3], conversely to infection with a low dose (102 CFU) [4]. On the other hand, $\gamma \delta \mathrm{T}$ cells are part of the innate immunity system and are excellent producers of IL-17 after antigen exposure. It has been shown that these cells, after low or high mycobacterial dose exposure, increase significantly the production of IL-17 at the site of infection, and this production is dependent of the presence of IL-23 that is mainly produced by DCs. Moreover, the presence of IL-17 is associated with enhanced neutrophil recruitment that is dependent of the presence of IL-23. Neutrophil plays a controversial role during Mycobacterium tuberculosis infection [5], this kind of innate immune cells are recruited during the first hours after exposure and posses several mechanism of mycobacterial contention and elimination; for example, the production of antimicrobial peptides, secreting cytokines and chemokines for $\mathrm{T}$ cell activation, recruitment and granulome formation. However, it is well known that high accumulation of neutrophils at the site of infection is related with a bad prognosis and increased lung immunopathology due to extensive tissue damage. Furthermore, multiple immunizations with M. bovis BCG after Mycobacterium tuberculosis exposure increase Th17 response and tissue damage that is dependent of neutrophil recruitment. Interestingly, the same degree of immune protection but without tissue damage was seen in mice deficient of IL-23p19 [2].

The role of IL-17-secreting T cells during immune response to mycobacterial challenge or molecules is now being elucidated. Khader SA [6] and co-workers showed that vaccination of mice with a 20 amino acid peptide derived from the $6 \mathrm{kDa}$ secreted protein ESAT- 6 , and restricted to I-Ab, induces IL-17 producing CD4+ T cells, which migrate to lungs after aerosol challenge with Mycobacterium tuberculosis H37Rv. Some chemokines such as CXCL9, CXCL10 and CXCL11 were expressed and are related with the accumulation of IL-17-producing T cells [6]. With these findings in mind, Chatterjee S [7] and co-workers, in an elegant work demonstrated that, ESAT- 6 promotes the induction of Th17 cells via interaction with TLR-2/MyD88 signaling in DCs. The lack of RD1 in M. tuberculosis and BCG resulted in no increase of TGF- $\beta$ and IL- 6 production, cytokines that are necessary for Th17 development; suggesting the importance of an intact RD1 region for an improved immune response [7].

In conclusion, although more in deep research is required for a real understanding of pathology related to Th17 immune response during tuberculosis, this knowledge must be taken into account for rational vaccine design.

\section{References}

1. Philips JA, Ernst JD (2012) Tuberculosis pathogenesis and immunity. Annu Rev Pathol 7: 353-384.

2. Torrado E, Cooper AM (2010) IL-17 and Th17 cells in tuberculosis. Cytokine Growth Factor Rev 21: 455-462.

3. Aujla SJ, Dubin PJ, Kolls JK (2007) Th17 cells and mucosal host defense. Semin Immunol 19: 377-382.

*Corresponding author: Mario Alberto Flores-Valdez, Center for Research and Assistance in Technology and Design of the State of Jalisco, AC, Medical and Pharmaceutical Biotechnology, Av normalistas 800, Col. Hills Normal, Guadalajara, Jalisco 44270, Mexico, E-mail: floresv@ciatej.net.mx,floresvz@yahoo.com

Received November 15, 2012; Accepted November 17, 2012; Published November 22, 2012

Citation: Pedroza-Roldán C, Barba J, Flores-Valdez MA (2012) Th17: A New Player to be Considered in Tuberculosis Studies. J Bacteriol Parasitol 3:e115. doi:10.4172/2155-9597.1000e115

Copyright: () 2012 Pedroza-Roldán C, et al. This is an open-access article distributed under the terms of the Creative Commons Attribution License, which permits unrestricted use, distribution, and reproduction in any medium, provided the original author and source are credited. 
Citation: Pedroza-Roldán C, Barba J, Flores-Valdez MA (2012) Th17: A New Player to be Considered in Tuberculosis Studies. J Bacteriol Parasitol 3:e115. doi:10.4172/2155-9597.1000e115

Page 2 of 2

4. Okamoto Yoshida Y, Umemura M, Yahagi A, O’Brien RL, Ikuta K, et al. (2010) Essential role of IL-17A in the formation of a mycobacterial infection-induced granuloma in the lung. $\mathrm{J}$ Immunol 184: 4414-4422.

5. Lowe DM, Redford PS, Wilkinson RJ, O'Garra A, Martineau AR (2012) Neutrophils in tuberculosis: friend or foe? Trends Immunol 33: 14-25.

6. Khader SA, Bell GK, Pearl JE, Fountain JJ, Rangel-Moreno J, et al. (2007)
IL-23 and IL-17 in the establishment of protective pulmonary CD4+ T cell responses after vaccination and during Mycobacterium tuberculosis challenge. Nat Immunol 8: 369-377.

7. Chatterjee S, Dwivedi VP, Singh Y, Siddiqui I, Sharma P, et al. (2011) Early secreted antigen ESAT- 6 of Mycobacterium tuberculosis promotes protective $\mathrm{T}$ helper 17 cell responses in a toll-like receptor-2-dependent manner. PLoS Pathog 7: e1002378. 